\title{
Molecular Diversity and Pathogenicity of Ralstonia solanacearum Species Complex Associated With Bacterial Wilt of Potato in Rwanda
}

\author{
Kalpana Sharma, ${ }^{1,2, \dagger}$ Jan Kreuze, ${ }^{1,3}$ Abdulwahab Abdurahman, ${ }^{1,2}$ Monica Parker, ${ }^{1,2}$ Anastase Nduwayezu, ${ }^{4}$ and Placide Rukundo ${ }^{4}$ \\ ${ }^{1}$ Consultative Group for International Agricultural Research (CGIAR) Research Program on Roots, Tubers, and Bananas (RTB) \\ ${ }^{2}$ International Potato Center (CIP), Sub-Saharan Africa Regional Office, Nairobi, Kenya \\ ${ }^{3}$ International Potato Center (CIP), Crop and Systems Sciences Division, Lima, Peru \\ ${ }^{4}$ Rwanda Agriculture and Animal Resources Development Board (RAB), Musanze Station, Northern Province, Rwanda
}

\begin{abstract}
Bacterial wilt (BW), caused by Ralstonia solanacearum species complex (RSSC), leads to substantial potato yield losses in Rwanda. Studies were conducted to (i) determine the molecular diversity of RSSC strains associated with BW of potato, (ii) generate an RSSC distribution map for epidemiological inferences, and (iii) test the pathogenicity of predominant RSSC phylotypes on six commercial potato cultivars. In surveys conducted in 2018 and 2019, tubers from wilting potato plants were collected for pathogen isolation. DNA was extracted from 95 presumptive RSSC strain colonies. The pathogen was phylotyped by multiplex PCR and typed at sequevar level. Phylotype II sequevar 1 strains were then haplotyped using multilocus tandem repeat sequence typing (TRST) schemes.

$(95.79 \%, n=91)$ and phylotype III $(4.21 \%, n=4)$. This is the first report of phylotype III strains from Rwanda. Phylotype II strains were identified as sequevar 1 and distributed across potato growing regions in the country. The TRST scheme identified 14 TRST haplotypes within the phylotype II sequevar 1 strains with moderate diversity index (HGDI $=0.55$ ). Mapping of TRST haplotypes revealed that a single TRST ' $8-5-12-7-5$ ' haplotype plays an important epidemiological role in BW of potato in Rwanda. None of the cultivars had complete resistance to the tested phylotypes; the level of susceptibility varied among cultivars. Cultivar Cruza, which is less susceptible to phylotype II and III strains, is recommended when planting potatoes in the fields with history of BW.
\end{abstract} Pathogenicity of one phylotype II strain and two phylotype III strains were tested on cultivars Kinigi, Kirundo, Victoria, Kazeneza, Twihaze, and Cruza. Two RSSC phylotypes were identified, phylotype II
Keywords: molecular epidemiology, multilocus tandem repeat sequence typing, RSSC, potato cultivars
Potato (Solanum tuberosum) is an important food security crop and a major source of household income for smallholder farmers in Rwanda. Potato grows well in the whole country from the highlands (major growing areas) to midlands (Kathiresan 2011). Nyabihu, Rubavu, Musanze, Burera, and Rutsiro districts in the northern and western provinces account for $79 \%$ of the total potato production in the country (NISR 2018). Due to the growing market demand, area under potato production in the northern, western, and southern provinces has increased substantially in recent years, including in some districts of the eastern province (NISR 2018). Despite the importance of potato in Rwanda, potato productivity remains at $9 \mathrm{tha}^{-1}$ (FAOSTAT 2017) largely due to bacterial wilt caused by Ralstonia solanacearum species complex (RSSC) (Muhinyuza et al. 2012; Uwamahoro et al. 2018).

$R$. solanacearum was originally described as a single species. Later phylogenetic analysis confirmed that it encompasses a highly heterogeneous group of bacteria belonging to several species and was subsequently classified as a species complex (Fegan and Prior 2005; Gillings and Fahy 1994; Guidot et al. 2007). RSSC strains fall

${ }^{\dagger}$ Corresponding author: K. Sharma; kalpana.sharma@cgiar.org

Funding: Funding support for this work was provided by the German Federal Ministry for Economic Cooperation and Development (BMZ) project number 16.7860.6-001.00 and contract number 81206684. Contribution for open access publishing from Bill and Melinda Gates Foundation is gratefully acknowledged.

*The $\boldsymbol{e}$-Xtra logo stands for "electronic extra" and indicates that two supplementary tables are published online.

The author(s) declare no conflict of interest.

Accepted for publication 21 July 2020.

Copyright $\odot 2021$ The Author(s). This is an open access article distributed under the CC BY-NC-ND 4.0 International license. into four genetically distinct phylotypes that correlate to strains' geographic origins: phylotype I (Asia), II (the Americas), III (Africa), and IV (Indonesia) (Fegan and Prior 2005; Poussier et al. 2000a, b). Subsequently, taxonomic revision of $R$. solanacearum led to the description of three species within the RSSC (Safni et al. 2014): (i) $R$. solanacearum, which includes all phylotype II strains, (ii) $R$. pseudosolanacearum with all phylotype I and phylotype III strains, and (iii) $R$. syzygi with all phylotype IV strains.

RSSC is ranked as the second most destructive bacterial plant pathogen after Pseudomonas syringae because of its wide geographical distribution and broad host range (Elphinstone 2005; Genin 2010; Mansfield et al. 2012). Direct economic impact of this pathogen is difficult to quantify precisely, but on potato alone it is responsible for an estimated loss over US\$1 billion each year worldwide, affecting more than 3 million farm families across 1.5 million ha in around 80 countries (Elphinstone 2005). In many countries where RSSC has quarantine status, important losses occur as a result of regulatory eradication measures and restrictions imposed on further production on contaminated land.

Rwanda has a very long history of bacterial wilt. Several studies indicate the serious challenges bacterial wilt poses to potato production (Butare 1987; Devaux et al. 1987; Elphinstone 2005; Lepoint and Maraite 2002; Mutimawurugo et al. 2019; Uwamahoro et al. 2018; Van der Zaag 1985). Losses due to RSSC on potato are estimated at 20 to $100 \%$, leading to huge economic losses and directly affecting food security and livelihoods of smallholder farmers (Mutimawurugo et al. 2019; Uwamahoro et al. 2018). More importantly, it is becoming a very serious impediment to the rapidly expanding potato production in Rwanda (Mutimawurugo et al. 2019).

RSSC diversity from potato has been described for many African countries (Abdurahman et al. 2017, 2019; Chesneau et al. 2018; Elphinstone 2005; Fegan and Prior 2005; Poussier et al. 2000b). In Ethiopia and Uganda, the most important epidemiological strains causing potato bacterial wilt were identified (Abdurahman et al. 2017, 2019). These strains with the capacity to overcome the resistance to bacterial wilt are rapidly spreading through the informal seed exchanges in potato growing regions within the country and between 
neighboring countries (Abdurahman et al. 2017, 2019). Based on studies of three isolates from Rwanda, Butare (1987) and Lepoint and Maraite (2002) reported the presence of biovar 2 and biovar 3 strains (phylotype II and I, respectively, based on Safni et al. (2014) classification), and biovar 3 strain being restricted to Kinigi Research station in Musanze district. Mutimawurugo et al. (2019) also identified three isolates collected from potatoes at Kinigi station, Musanze as biovar 3 (phylotype I). A phylotype II sequevar 1 (biovar 2) strain (JT573) was also reported from potato in Rwanda (Poussier et al. 2000a, b). Despite the long history of the presence of $R$. solanacearum in Rwanda, there are no detailed reports available on the genetic diversity of RSSC and their geographical distribution in the country.

Disease management remains limited and is hampered by the ability of RSSC to survive in wet environments, on plant debris, or in asymptomatic weed hosts, which act as inoculum reservoirs (Álvarez et al. 2008; Elphinstone 2005; Mansfield et al. 2012; van Overbeek et al. 2004). In the absence of effective chemical control methods, prevention of bacterial wilt largely relies on the availability of RSSC free planting materials, crop rotation, and strict phytosanitary measures combined with the use of tolerant potato varieties.

Tolerant and resistant potato varieties can greatly reduce bacterial wilt development and buildup of soilborne inoculum in fields (Elphinstone 2005; Mansfield et al. 2012); however, development of bacterial wilt resistant potato varieties is complicated and hampered by strong host-pathogen-environment interactions (Genin 2010). Phylotype composition of RSSC can shift rapidly in response to selection pressure (Wicker et al. 2012) and occurrence of more than one phylotype in one field or location can break down the genetic resistance quickly (French et al. 1998; Ravelomanantsoa et al. 2018). Potatoes can be infected by all four phylotype strains; cultivars like Cruza and Molinera have shown some degree of bacterial wilt tolerance in East Africa, while others have demonstrated differential susceptibility (French et al. 1998; Muthoni et al. 2014). Recently, the reaction of nine potato cultivars to a Rwandan RSSC phylotype II strain were assessed under greenhouse conditions (Uwamahoro et al. 2020). However, the reaction of commonly grown potato cultivars to the predominant RSSC phylotypes of Rwanda is not known.

Smallholder farmers in Rwanda are unable to implement effective crop rotation practices. This is further complicated by the informal potato seed system (Mpyisi et al. 2003; Uwamahoro et al. 2018). The formal seed system supplies only $5 \%$ of the national seed demand (Demo et al. 2015), and lack of certified potato seed contributes to further distribution of RSSC via latently infected tubers (Hayward 1974; Janse 1996). The informal seed system and unrestricted seed movement within Rwanda and along the borders with neighboring countries (Demo et al. 2015) pose a clear threat in disseminating RSSC to uninfected regions and may introduce new strains. It is therefore important to have a comprehensive understanding of strain type being disseminated along with the seed movement and patterns of disease spread to design effective management strategies.

Multilocus variable-number tandem-repeat (VNTR) analysis (MLVA) based typing methods, a classic length-based MLVA analysis method (Ravelomanantsoa et al. 2018), and a tandem repeat sequence typing (TRST) method (Abdurahman et al. 2017, 2019; N'Guessan et al. 2013; Parkinson et al. 2013) have been efficient for routine typing and for investigating the genetic structures of the RSSC strains, their geographical distribution, and the relationship among closely related RSSC strains belonging to the same sequevar. The classic length-based MLVA typing method uses amplicon sizebased genotyping to type the strain, whereas TRST uses the sequence-based VNTR analysis typing method. The tandem repeats (TR) loci are among the most variable regions in bacterial genomes (Davey et al. 2011) and therefore have the potential to resolve the genetic diversity of monomorphic or monoclonal pathogens (van Belkum et al. 1998). Recently, the TRST technique based on the VNTR loci and primers of N'Guessan et al. (2013) and Parkinson et al. (2013) was employed to trace the bacterial wilt epidemic strains in seed potato production in Ethiopia (Abdurahman et al. 2017), and to map the most common RSSC strains along with seed potato movement in Uganda (Abdurahman et al. 2019).

The present study was undertaken to characterize the molecular diversity of RSSC strains isolated from major potato growing regions of Rwanda, to generate an RSSC distribution map that may provide epidemiological inferences to develop future bacterial wilt management interventions, and to test potato cultivars commonly grown in Rwanda for their reactions to the predominant RSSC phylotypes in the country.

\section{Materials and Methods}

Survey districts and sampling of potato tubers. Surveys were carried out during the long and short rain seasons of 2018 and 2019 in the major potato growing regions of the country (Table 1). The survey sites were selected by the team of RAB, Musanze station, northern province, Rwanda. The 2018 survey covered 12 districts in four major potato production administrative zones of Rwanda (NISR 2018): Nyamagabe and Nyarugu in the southern province; Musanze and Burera in the northern province; Karongi, Nyabihu, Rubavu, and Rutsiro in the western province; and Ngoma, Rwamagana, Kyonza, and Gatsibo districts in the eastern province. One hundred and four potato samples from 12 districts were collected in the 2018 survey and 49 samples from five districts (Burera, Gakenke, Rulindo, Musanze, and Nyabihu) in 2019. Five wilting potato hills were randomly selected per potato field, and up to two tubers were sampled per hill (10 tubers per potato field). Tubers were wrapped in a paper towel and put in a labeled paper bag and kept at room temperature until processing for bacterial isolation. To prevent the spread of the pathogen between surveyed potato fields, boots were sprayed with $5 \%$ sodium hypochlorite solution and hands disinfected with $70 \%$ ethanol while changing gloves between handling samples. The number of samples per district was based on the area under potato production in the respective districts. For sampling, potato fields at least $2 \mathrm{~km}$ apart were closely inspected for typical bacterial wilt symptoms and tuber samples from the symptomatic plants were collected for the pathogen isolation. Sample handling, washing, and processing of the potato tuber vascular tissue for the bacterial suspension were as described in Abdurahman et al. (2017).

Isolation of bacteria and DNA extraction. Bacterial suspensions extracted from potato tuber vascular tissue was streak plated on semiselective media from South Africa (SMSA) and incubated at $28^{\circ} \mathrm{C}$ for 48 to $72 \mathrm{~h}$ (Abdurahman et al. 2017). Presumptive RSSC colonies from SMSA media were then plated on casamino acid peptone glucose (CPG) agar media and incubated at $28^{\circ} \mathrm{C}$ for $24 \mathrm{~h}$ to confirm the typical Ralstonia colonies. A total of 95 (62 from 2018 and 33 from 2019) presumptive RSSC colonies were obtained (Table 1). A loopful of presumptive RSSC single colonies from a CPG plate was then suspended in $100 \mu \mathrm{l}$ molecular biology grade water and boiled at $95^{\circ} \mathrm{C}$ for $15 \mathrm{~min}$ on a thermal cycler for DNA extraction (Weller et al. 2000).

PCR detection of RSSC and phylotype identification. DNAs from the boiled presumptive Ralstonia isolates were first screened using RSSC-specific primer pairs 759/760 of Opina et al. (1997) (Supplementary Table S1). Phylotyping was done by multiplex PCR combining the four phylotype-specific primers of Fegan and Prior (2005) using AccuPower Taq PCR Master Mix (Bioneer, Korea). The cycling conditions for PCR were: initial denaturation of $15 \mathrm{~min}$ at $95^{\circ} \mathrm{C}$, then 30 cycles of $30 \mathrm{~s}$ at $94^{\circ} \mathrm{C}, 1 \mathrm{~min}$ at $59^{\circ} \mathrm{C}$, $1 \mathrm{~min}$ at $72^{\circ} \mathrm{C}$, and final extension of $10 \mathrm{~min}$ at $72^{\circ} \mathrm{C}$. The PCR reaction reagents, volumes, and protocol were as described by Abdurahman et al. (2019).

Sequevar typing. A total of 95 isolates were processed for sequevar typing. A partial fragment of virulence-associated mega-plasmid endoglucanase ( $\mathrm{egl}$ ) gene was amplified from genomic DNA using Endo-F and Endo-R primers of Fegan and Prior (2005). The PCR protocol, sequencing primers, and alignment processes were as described by Abdurahman et al. (2019) except that AccuPower Taq PCR Master Mix (Bioneer, Korea) was used instead of Fermentas Master mix. Cleaned PCR products were sequenced at Macrogen Europe B.V. (Amsterdam, the Netherlands) using the same Endo-F 
and Endo-R primers. All isolates that clustered with phylotype II sequevar 1 reference strains were confirmed by individually running the egl sequences in the typing computer program of Stulberg and Huang (2016). Ninety-five sequences that were typed as sequevar 1 , and those with RSSC characteristics were aligned in bioedit software using the CLUSTALW algorithm along with selected reference strains pooled from the NCBI database and clipped at start (ACGGAC) and stop (CAGTGG) sequences. The aligned sequences were used to generate a neighbor-joining phylogenetic tree with 1,000 bootstrap resampling using MEGA6 software (Tamura et al. 2013).

Tandem repeat sequence typing (TRST). Sequence typing of the alleles of four VNTR loci (L504, L539, L540, L563) described by Parkinson et al. (2013), and locus IPO100 described by N'Guessan et al. (2013) were selected to distinguish $R$. solanacearum phylotype II sequevar 1 strains $(n=91)$. PCR reaction and cycling conditions for all the loci were as described in Abdurahman et al. (2017, 2019). PCR mix (2×, AccuPower Taq PCR Master Mix, Bioneer, Korea) was used. The amplified PCR products were run on $1.5 \%$ agarose gel stained with $1 \times$ GelRed (Biotium) and the target amplicon was cut under UV light and immediately cleaned with Wizard SD gel and PCR clean-up system (Promega) following the kit protocol, and sequenced using sequencing primers described by Parkinson et al. (2013) and the forward primer of N'Guessan et al. (2013) for locus IPO100 at Macrogen Europe. Tandem repeat sequences were counted manually after aligning the sequences with the start and finish sequence definitions by Parkinson et al. (2013) using ClustalW alignment tool in BioEdit software. Postrepeat sequence (GGTGC(C/T) CCGGTGCCCGGTGCCCAGTGCTCAGTGCTCAGTGCCCGGT GATTGCC) for locus IPO100 was used to define the end of tandem repeat sequence. A string of tandem repeat numbers in the order of L504-L539-L540-L563-IPO100 represents a TRST haplotype (a haplotype here being isolates with identical TRST profiles) (Supplementary Table S2).

Pathogen. For the pathogenicity test, three RSSC strains, Rw7 (phylotype II sequevar 1), Rw15 (phylotype III), and Rw62 (phylotype III) collected from infected tubers grown in northern, southern, and western provinces of Rwanda, respectively, were used. Rw7 was selected because it belongs to TRST ' $8-5-12-7-5$ ' haplotype profile with the widest distribution in the northern, western, and southern provinces, whereas Rw15 (phylotype III) and Rw62 (phylotype III) were selected based on their local importance in northern and eastern provinces. Ralstonia strains were grown at $30^{\circ} \mathrm{C}$ on CPG medium. Bacterial cells were harvested in sterile distilled water from 48 -hgrown culture plates. Bacterial concentration was determined by measuring their optical density $(600 \mathrm{~nm})$ and adjusted to $5 \times 10^{8}$ $\mathrm{CFU} \mathrm{ml} \mathrm{m}^{-1}$. Freshly prepared inoculum was used for inoculation.

Cultivation and inoculation of plants. The pathogenicity of Rw7, Rw15, and Rw62 was tested on six commercial potato cultivars: Kinigi (CIP378699.2), Kirundo, Victoria (CIP381381.20), Kazeneza (CIP393077.159), Twihaze (CIP393371.58), and Cruza (CIP720118). Cultivars Kinigi, Kirundo, Cruza, and Victoria were released in 1980s and are commonly grown by smallholder farmers in Rwanda, whereas cultivars Kazeneza and Twihaze, released in 2018 , are being promoted for production by RAB in major potato producing districts of the country. Sprouted tubers were grown in plastic pots $(5 \times 7 \mathrm{~cm}$, one tuber per pot $)$ filled with steamsterilized soil. Plants were maintained in a growth room (biosafety level 2 at Biosciences eastern and central Africa (BecA)-ILRI Hub, Nairobi, Kenya) at 18 and $25 \pm 2{ }^{\circ} \mathrm{C}$ (night and day temperature, respectively) with $70 \%$ relative humidity and a 12 -h photoperiod. Plants were watered daily except for one day prior to inoculation and fertilized weekly with $5 \mathrm{ml}$ of mineral fertilizer $(80 \mathrm{~g}$ of 15:15: 18 NPK fertilizer per liter stock solution, $5 \mathrm{ml}$ stock solution per liter fertilizer solution).

Five-day-old plants were inoculated by pipetting $10 \mathrm{ml}$ of bacterial suspension containing $5 \times 10^{8} \mathrm{CFU} \mathrm{ml}^{-1}$ onto the soil surface at the base of each plant at 20 days after planting. Control plants were inoculated with $10 \mathrm{ml}$ sterile water. To avoid cross-contamination, the plants in each strain treatment (plus the noninoculated control) were kept in separate trays. The trial was arranged in a randomized complete block design with five replicates (five plants per replication per strain), and the trial was repeated once.

Disease development and severity. Inoculated plants were monitored daily to record days of disease onset. Bacterial wilt incidence and severity were visually assessed on individual plants at 4-day intervals starting 4 days after inoculation (DAI) to a maximum of 44 DAI. Bacterial wilt severity was rated using a 0 to 4 scale to separate the wilting into classes, where $0=$ no wilting, $1=1$ to $25 \%$ wilting foliage, $2=26$ to $50 \%$ wilting foliage, $3=51$ to $75 \%$ wilting foliage, and $4=76$ to $100 \%$ wilting foliage or dead (Horita and Tsuchiya 2001). A bacterial wilt disease severity index (DSI) was calculated using the equation of Strelkov et al. (2006): DSI $=\{\Sigma[$ (class number $) \times$

Table 1. Potato survey by province and district with altitude range covered, number of samples collected and processed, and the number of Ralstonia solanacearum species complex (RSSC) isolates per district from 2018 and 2019 surveys in Rwanda

\begin{tabular}{|c|c|c|c|c|c|c|c|c|}
\hline \multirow[b]{2}{*}{ Province } & \multirow[b]{2}{*}{ District } & \multirow[b]{2}{*}{ Elevation range (masl) ${ }^{\mathbf{a}}$} & \multicolumn{2}{|c|}{$\begin{array}{l}\text { Number of } \\
\text { samples } \\
\text { collected and } \\
\text { processed }\end{array}$} & \multirow{2}{*}{$\begin{array}{l}\text { Total number } \\
\text { of samples collected } \\
\text { and processed }\end{array}$} & \multicolumn{2}{|c|}{$\begin{array}{c}\text { Confirmed } \\
\text { RSSC strains }\end{array}$} & \multirow{2}{*}{$\begin{array}{c}\text { Total number } \\
\text { of confirmed } \\
\text { RSSC strains }\end{array}$} \\
\hline & & & 2018 & 2019 & & 2018 & 2019 & \\
\hline \multirow[t]{4}{*}{ Northern } & Burera & $1,866-2,281$ & 22 & 11 & 33 & 13 & 6 & 19 \\
\hline & Gakenke & $1,820-2,001$ & $\mathrm{NA}^{\mathrm{c}}$ & 9 & 9 & NA & 8 & 8 \\
\hline & Rulindo & $1,854-2,260$ & NA & 16 & 16 & NA & 14 & 14 \\
\hline & Musanze & $1,848-2,558$ & 20 & 9 & 29 & 10 & 5 & 15 \\
\hline \multirow[t]{4}{*}{ Eastern } & Gatsibo & 1,521 & 1 & NA & 1 & 0 & NA & 0 \\
\hline & Ngoma & $1,652-1,653$ & 3 & NA & 3 & 1 & NA & 1 \\
\hline & Kayonza & $1,452-1,571$ & 3 & NA & 3 & 2 & NA & 2 \\
\hline & Rwamagana & $1,528-1,529$ & 4 & NA & 4 & 1 & NA & 1 \\
\hline \multirow[t]{4}{*}{ Western } & Karongi & $2,319-2,561$ & 6 & NA & 6 & 5 & NA & 5 \\
\hline & Nyabihu & $2,322-2,467$ & 14 & 4 & 18 & 7 & 0 & 7 \\
\hline & Rubavu & $2,016-2,368$ & 13 & NA & 13 & 8 & NA & 8 \\
\hline & Rutsiro & 2,266 & 1 & NA & 1 & 1 & NA & 1 \\
\hline \multirow[t]{2}{*}{ Southern } & Nyamagabe & $2,051-2,399$ & 12 & NA & 12 & 11 & NA & 11 \\
\hline & Nyaruguru & $1,898-2,345$ & 5 & NA & 5 & 3 & NA & 3 \\
\hline Total & & & 104 & 49 & 153 & 62 & 33 & $95^{\mathrm{d}}$ \\
\hline
\end{tabular}

${ }^{a}$ Meters above sea level.

b A total of 153 processed samples from 2018 and 2019 surveys.

${ }^{\mathrm{c}} \mathrm{NA}=$ Not available; samples were not collected because there were no potato crops in the field on that survey year.

d Out of 153 processed samples, only 95 were pure culture RSSC strains. 
(number of plants in each class)] / [(total number plants per sample) $\times$ (number of classes -1$)]\} \times 100$. Completely or partially wilted plants were tagged to avoid double counting in subsequent assessments and to avoid the possibility of missing those that die early during the experiment. Bacterial wilt incidence (BWI) was calculated as the proportion of inoculated plants that showed the typical symptoms of bacterial wilt. Area under bacterial wilt severity progress curve (AUDPC) was calculated from the bacterial wilt severity index value from 4 DAI to 24 DAI (Campbell and Madden 1990) to compare disease evolution among treatments.

Asymptomatic plants were sampled and analyzed for latent infection by RSSC strains at 44 DAI. About $1 \mathrm{~cm}$ long stem base of the plants was cut and manually crushed in $2 \mathrm{ml}$ of extraction buffer (phosphate buffer $\mathrm{pH}$ 7.0) containing antioxidant tetrasodium pyrophosphate $\left(1 \mathrm{~g} \mathrm{liter}^{-1}\right)$ into a maceration bag (Abdurahman et al. 2017). After maceration, the samples were left to stand for $15 \mathrm{~min}, 1.5 \mathrm{ml}$ of suspension was transferred to $2 \mathrm{ml}$ centrifuge tubes for bacterial concentration by centrifugation as described above, and the concentrated pellet was immediately resuspended in phosphate buffer ( $\mathrm{pH}$ 7.0). The resuspended pellet was used for streak plating on semiselective agar media (SMSA) and incubated for $48 \mathrm{~h}$ at $28^{\circ} \mathrm{C}$. Presumptive Ralstonia single colonies selected from SMSA media plates were then streak plated on CPG agar media and incubated for $24 \mathrm{~h}$ at $28^{\circ} \mathrm{C}$. Asymptomatic plants were scored positive for latent infection when characteristic Ralstonia colonies were unequivocally observed on the plates. DNA was extracted from fresh colonies grown on CPG agar media overnight as described above, and then identified at the RSSC phylotype level by the phylotyping scheme of Fegan and Prior (2005) using PCR with $759 / 760$ primer pairs of Opina et al. (1997) to confirm the causal agent.

Data analysis. Allelic diversity at each locus was calculated using the unbiased estimator of Nei (1978): $h=1-\sum x_{i}^{2}[n /(n-1)]$, where $x_{i}$ is the frequency of the $\mathrm{i}^{\text {th }}$ allele at the locus, $\mathrm{n}$ is the number of isolates, and $\mathrm{n} /(\mathrm{n}-1)$ is a correction for bias in small samples (Nei 1978; Selander et al. 1986). The analysis was performed using built-in algorithm in the LIAN Linkage Analysis online (http://guanine. evolbio.mpg.de/cgi-bin/lian/lian.cgi.pl). The overall discriminatory power of the TRST scheme was tested with the HunterGaston index of diversity (HGDI; Hunter and Gaston 1988) by using an online tool (http://insilico.ehu.es/mini_tools/discriminatory_power/index.php). The relationship between strains was analyzed using eBURST v3 to define profiles and clonal clusters using default settings. The standardized index of association $\left(I_{\mathrm{A}}^{\mathrm{S}}\right)$ between different alleles of the loci was calculated using LIAN Linkage Analysis (Haubold and Hudson 2000) set to 100,000 Monte Carlo (MC) simulations to test the null hypothesis of linkage equilibrium $\left(I_{\mathrm{A}}^{\mathrm{S}}=0\right)$. Only samples presenting complete TRST haplotype profile were included in the data analysis.

Treatment effects of the pathogenicity trial were assessed using the mixed model analysis of variance of the data with the cultivar as the fixed effect and replication as a random effect (PROC MIXED, SAS software version 9.11; SAS Institute Inc., Cary, NC). Data for each trial were tested for normality using the Shapiro-Wilk test of residuals and checked for outliers using Lund's test of standardized residuals (Lund 1975). No outliers were found in any data set. There was no effect of repetition or repetition-treatment; therefore, the data were pooled across repetition for analysis. Means were separated using Tukey's test. Prior to analysis, percent data were arcsinetransformed when necessary to improve the normality and homogeneity of variance, but nontransformed means are presented for uniformity of presentation. Differences were significant as $P \leq$ 0.05 unless otherwise noted.

\section{Results}

Isolation, detection, phylotype, and sequevar identification. Out of 153 samples processed for bacterial isolation, only 95 samples were identified as RSSC strains with $759 / 760$ primers (Table 2). The phylotyping of these strains classified them as phylotype II $R$. solanacearum $(n=91)$ and phylotype III $R$. pseudosolanacearum $(\mathrm{Rs})$ ( $n=4$; Rw15, Rw49, Rw61, and Rw62). The percentage of the confirmed RSSC strains was highest in the northern province ( $n=56$; phylotype II $=57.89 \%$, phylotype III $=1.05 \%)$, followed by the western $(n=21$; phylotype II $=22.11 \%)$, the southern $(n=14$; phylotype II $=13.68 \%$, phylotype III $=1.05 \%)$, and the eastern $(n=4$; phylotype II $=2.11 \%$, phylotype III $=2.11 \%$ ). Of 55 phylotype II strains isolated from the northern province, 19 were from Burera district, 14 each from Musanze and Rulindo, and eight from Gakenke. The percentage of phylotype II strains in the western province ranged from 1.05 to 8.42 among Rubavu, Nyabihu, Karongi, and Rutsiro districts. Nyamagabe and Nyaruguru districts of the southern province had $11.58 \%$ and $2.11 \%$ phylotype II strains, respectively. Similarly, in the eastern province, phylotype II strains remained at $1.05 \%$ each in Ngoma and Rwamagana districts. Kayonza district of the eastern province is the only district represented by two phylotype III strains (Rw61 and Rw62).

Table 2. Ralstonia solanacearum species complex (RSSC) with their respective phylotype, number of strains, and sequevar with the strain ID per district from 2018 and 2019 surveys in Rwanda

\begin{tabular}{|c|c|c|c|c|}
\hline Province & District & RSSC phylotype & Number of strains & Sequevar (strain ID) \\
\hline \multirow[t]{5}{*}{ Northern } & Burera & II & 19 & $\begin{array}{l}1 \text { (Rw27, Rw28, Rw29, Rw30, Rw31, Rw32, Rw33, Rw34, Rw35, Rw36, Rw37, Rw38, } \\
\text { Rw39, Rw82, Rw83, Rw84, Rw85, Rw86, Rw87) }\end{array}$ \\
\hline & Gakenke & II & 8 & 1 (Rw88, Rw89, Rw90, Rw91, Rw92, Rw93, Rw94, Rw95) \\
\hline & Musanze & II & 14 & $\begin{array}{l}1 \text { (Rw6, Rw7, Rw8, Rw9, Rw10, Rw11, Rw12, Rw13, Rw14, Rw63, Rw64, Rw65, Rw66, } \\
\text { Rw67) }\end{array}$ \\
\hline & & III & 1 & $\mathrm{ND}^{\mathrm{a}}(\mathrm{Rw} 15)$ \\
\hline & Rulindo & II & 14 & $\begin{array}{l}1 \text { (Rw68, Rw69, Rw70, Rw71, Rw72, Rw73, Rw74, Rw75, Rw76, Rw77, Rw78, Rw79, } \\
\text { Rw80, Rw81) }\end{array}$ \\
\hline \multirow[t]{4}{*}{ Western } & Karongi & II & 5 & 1 (Rw1, Rw2, Rw3, Rw4, Rw5) \\
\hline & Nyabihu & II & 7 & 1 (Rw40, Rw41, Rw42, Rw43, Rw44, Rw45, Rw47) \\
\hline & Rubavu & II & 8 & 1 (Rw46, Rw51, Rw52, Rw53, Rw54, Rw55, Rw56, Rw57) \\
\hline & Rutsiro & II & 1 & 1 (Rw59) \\
\hline \multirow[t]{3}{*}{ Southern } & Nyamagabe & II & 11 & 1 (Rw16, Rw17, Rw18, Rw19, Rw20, Rw21, Rw22, Rw23, Rw24, Rw25, Rw26) \\
\hline & Nyaruguru & II & 2 & 1 (Rw48, Rw50) \\
\hline & & III & 1 & 29 (Rw49) \\
\hline \multirow[t]{3}{*}{ Eastern } & Kayonza & III & 2 & 21 (Rw61), NDb (Rw62) \\
\hline & Ngoma & II & 1 & 1 (Rw58) \\
\hline & Rwamagana & II & 1 & 1 (Rw60) \\
\hline Total ${ }^{\mathrm{c}}$ & & & 95 & \\
\hline
\end{tabular}

a Not determined, not tested.

${ }^{\mathrm{b}}$ Not determined, likely a new sequevar not yet named.

${ }^{c}$ Out of 95 RSSC strains, 91 strains were Phylotype II and four were Phylotype III. 


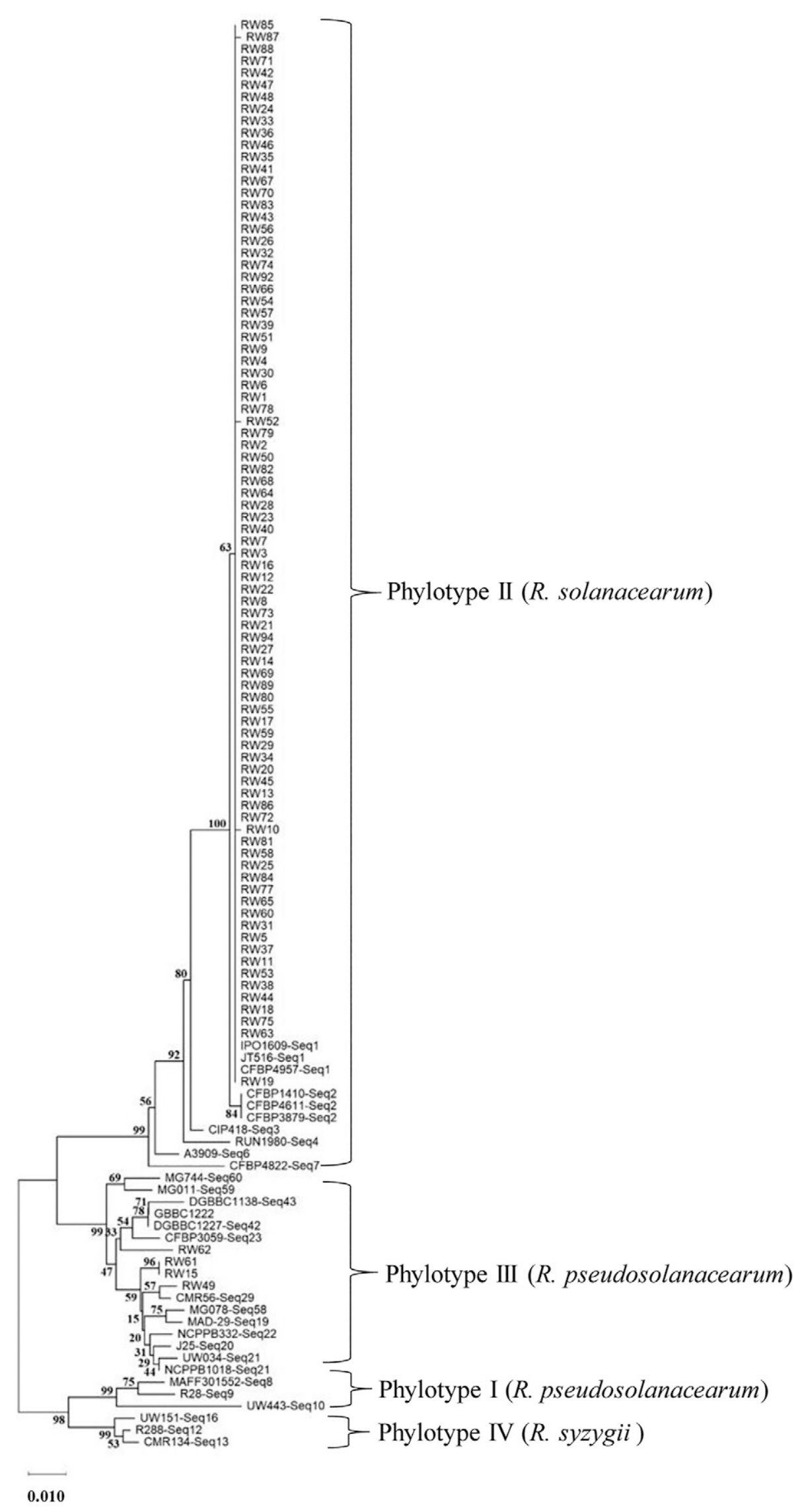

Fig. 1. Phylogenetic tree of partial egl gene sequences of 91 phylotype II Ralstonia solanacearum and four phylotype III R. pseudosolanacearum strains from Rwanda in 2018 and 2019 , and 29 reference strains from the NCBI database. The strains have a prefix 'RW', and RW with different serial numbers indicates different strains. The evolutionary history was inferred using the neighbor-joining method. The percentage of replicate trees in which the associated taxa clustered together in the bootstrap test (1,000 replicates) are shown next to the branches.

774 Plant Disease / Vol. 105 No. 4 
All the phylotype II strains clustered with sequevar 1 reference strains IPO1609 and JT516 by neighbor-joining phylogenetic analysis of the $e g l$ gene sequences identifying them as sequevar 1 strains, which were also confirmed by the typing program of Stulberg and Huang (2016) (Fig. 1). Phylotype III Rw49 strain showed 99\% sequence identity with sequevar 29 (CRM56, CIP358, CFBP6942), whereas phylotype III Rw15 and Rw61 strains showed 99\% sequence identity with strain NCPPB 1018 , but $<99 \%$ to strain UW034 both belonging to sequevar 21 when aligned. Considering the $99 \%$ sequence identity criteria set by Fegan and Prior (2005) for sequevar identity, the result was thus ambiguous and also not supported by phylogenetic analysis, which grouped Rw15 and Rw61 in a separate cluster from sequevar 21 strain (Fig. 1). Phylotype III Rw62 strain had no hit for $99 \%$ sequence identity in GenBank and had no clear cluster on the phylogenetic tree with specific sequevar, indicating that it is possibly a new sequevar.

Multilocus tandem repeat sequence typing. Five VNTR loci (L504, L539, L540, L563, and IPO100) of all 91 phylotype II sequevar 1 strains were sequenced for TRST analysis. Out of 91 phylotype II sequevar 1 strains, only 73 strains had successful sequences in all the loci that identified 14 TRST haplotypes (Table 3). TRST '8-5-127-5' haplotype $(n=50)$ was the most frequent followed by ' $8-5-13-7-$ 5', '8-5-12-6-5', and '8-4-12-7-5' $(n=3)$. Haplotypes '8-6-12-7-5', '8-5-12-8-5', '8-5-12-7-6', '8-5-12-7-6', and '8-5-12-7-4' were each represented by two strains, whereas ' $9-5-12-7-5$ ', ' $8-5-11-7-5$ ', ' $8-5$ 11-6-5', '8-3-12-7-5', '7-5-13-7-5', and '7-5-12-7-5' haplotypes were represented by a single strain.

Allelic diversity, discriminatory power, and linkage disequilibrium. Locus L539 had the highest allelic diversity $(h=0.180)$ followed by L540 and L563 ( $h=0.179$ ), but both loci were poorly discriminatory, according to the classification of Sola et al. (2003). The analysis of HGDI showed moderate discriminatory power $($ HGDI $=0.55)$ of the TRST scheme on Rwandan phylotype II sequevar 1 strains analyzed in this study (Table 4). The parametric (para) quantitative equilibrium test together with Monte Carlo simulation model for the standardized index of association of the alleles from the five VNTR loci were statistically significant in the LIAN 3.7 analysis $\left(I_{\mathrm{A}}^{\mathrm{S}}=\right.$ $0.1199, P_{\text {para }}=2.40 \times 10^{-3}, P_{\mathrm{MC}}=7.29 \times 10^{-7}$ ), indicating a nonclonal population structure of sequevar 1 strains from Rwanda.

Phylogeography of TRST haplotypes. The northern province was represented by 13 TRST haplotypes ('7-5-12-7-5', '7-5-13-75', '8-3-12-7-5', '8-4-12-7-5', '8-5-11-6-5', '8-5-11-7-5', '8-5-126-5', '8-5-12-7-5', '8-5-12-7-6', '8-5-12-8-5', '8-5-13-7-5', '8-6$12-7-5$ ', and ' $9-5-12-7-5$ '), southern province by four haplotypes ('8-5-12-7-5', '8-5-12-7-6', '8-5-12-8-5', and '8-6-12-7-5'), western by two haplotypes (' $8-5-12-7-4$ ' and ' $8-5-12-7-5$ '), and eastern by a single haplotype ('8-5-13-7-5') (Table 3). TRST '8-5-12-7-5' haplotype had the widest distribution being represented in nine out of the 14 surveyed districts in the northern $(n=29$ : Burera $=13$, Musanze $=$ 9, Rulindo $=5$, and Gakenke $=2)$, western $(n=13$ : Nyabihu $=6$, Karongi $=5$, Rubavu $=1$, and Rutsiro $=1)$, and southern provinces $(n=8$ : Nyamagabe $=8$ ). This was followed by TRST ' $8-5-13-7-5$ ' haplotype being represented in three districts, one each in Musanze district of the northern, and Ngoma and Rwamagana districts of the eastern provinces.

Mapping of the most frequent TRST haplotype of phylotype II sequevar 1 strains to the respective districts with the dominant seed distribution patterns from the northern to western, southern, and eastern provinces, it became apparent that $R$. solanacearum phylotype II strains with TRST ' $8-5-12-7-5$ ' haplotype was cross-regionally more important in Rwanda (Fig. 2). Similarly, TRST '8-5-12-7-6', '8-512-8-5', and ' $8-6-12-7-5$ ' haplotypes were identified from the northern and southern provinces, whereas TRST '8-5-13-7-5' haplotype was restricted to Musanze district of the northern, and Ngoma and Rwamagana districts of the eastern provinces.

Pathogenicity testing. Symptoms on the inoculated plants were progressive wilting from the young leaves, followed by complete wilting over time. Bacterial wilt incidence and severity were observed for all strains and cultivar combinations, but the symptoms developed earlier and were more severe on plants inoculated with Rw7, followed by Rw15, and least with Rw62 (Fig. 3). Substantial levels of infection were first observed at $4 \mathrm{DAI}$ in plants for Rw7 (up to 77\% BWI), at 8 DAI in plants for Rw15 (up to $84 \%$ BWI), and at 40 DAI in plants for Rw62 (up to $30 \%$ BWI). Increase in incidence and severity was roughly linear over time but occurred at different rates for different strains and cultivars. Cultivars Victoria, Kinigi, and Kirundo

Table 4. Range of tandem repeat counts, number of alleles, allelic diversity of variable number tandem repeat (VNTR) loci, and overall discriminatory power of tandem repeat sequence typing (TRST) schemes for 73 phylotype II Ralstonia solanacearum sequevar 1 strains from 2018 and 2019 surveys in Rwanda

\begin{tabular}{|c|c|c|c|c|}
\hline $\begin{array}{l}\text { Locus } \\
\text { name }\end{array}$ & $\begin{array}{c}\text { Range of } \\
\text { tandem repeat } \\
\text { counts }\end{array}$ & $\begin{array}{l}\text { Number } \\
\text { of alleles }\end{array}$ & $\begin{array}{c}\text { Allelic } \\
\text { diversity } \\
\text { (H) }\end{array}$ & $\begin{array}{c}\text { Hunter-Gatson index } \\
\text { of diversity (HGDI) }\end{array}$ \\
\hline L504 & $8-9$ & 2 & 0.106 & $0.5468^{a}$ \\
\hline L539 & $3-6$ & 4 & 0.180 & \\
\hline L540 & $11-13$ & 3 & 0.179 & \\
\hline L563 & $6-8$ & 3 & 0.179 & \\
\hline IPO100 & $4-6$ & 3 & 0.155 & \\
\hline
\end{tabular}

a Moderate HGDI.

Table 3. Summary of the multilocus tandem repeat sequence typing (TRST) haplotype profile of 73 phylotype II Ralstonia solanacearum sequevar 1 strains from the surveyed districts of the northern, western, southern, and eastern provinces of Rwanda in 2018 and 2019

\begin{tabular}{|c|c|c|c|c|c|c|c|c|c|c|c|c|c|}
\hline \multirow{2}{*}{$\begin{array}{l}\text { TRST } \\
\text { haplotype }\end{array}$} & \multicolumn{4}{|c|}{ Northern $^{a}$} & \multicolumn{4}{|c|}{ Western $^{\mathbf{a}}$} & \multicolumn{2}{|c|}{ Southern $^{a}$} & \multicolumn{2}{|c|}{ Eastern $^{a}$} & \multirow[b]{2}{*}{ Total $^{\text {b }}$} \\
\hline & Burera & Musanze & Rulindo & Gakenke & Nyabihu & Karongi & Rubavu & Rutsiro & Nyamagabe & Nyaruguru & Ngoma & Rwamagana & \\
\hline $8-5-12-7-5$ & 13 & 9 & 5 & 2 & 6 & 5 & 1 & 1 & 8 & 0 & 0 & 0 & 50 \\
\hline $8-5-13-7-5$ & 0 & 1 & 0 & 0 & 0 & 0 & 0 & 0 & 0 & 0 & 1 & 1 & 3 \\
\hline $8-4-12-7-5$ & 0 & 0 & 1 & 2 & 0 & 0 & 0 & 0 & 0 & 0 & 0 & 0 & 3 \\
\hline $8-5-12-6-5$ & 3 & 0 & 0 & 0 & 0 & 0 & 0 & 0 & 0 & 0 & 0 & 0 & 3 \\
\hline $8-5-12-7-4$ & 0 & 0 & 0 & 0 & 0 & 0 & 2 & 0 & 0 & 0 & 0 & 0 & 2 \\
\hline $8-5-12-7-6$ & 1 & 0 & 0 & 0 & 0 & 0 & 0 & 0 & 1 & 0 & 0 & 0 & 2 \\
\hline $8-5-12-8-5$ & 0 & 1 & 0 & 0 & 0 & 0 & 0 & 0 & 1 & 0 & 0 & 0 & 2 \\
\hline $8-6-12-7-5$ & 0 & 0 & 1 & 0 & 0 & 0 & 0 & 0 & 0 & 1 & 0 & 0 & 2 \\
\hline $7-5-12-7-5$ & 0 & 0 & 0 & 1 & 0 & 0 & 0 & 0 & 0 & 0 & 0 & 0 & 1 \\
\hline $7-5-13-7-5$ & 1 & 0 & 0 & 0 & 0 & 0 & 0 & 0 & 0 & 0 & 0 & 0 & 1 \\
\hline $8-3-12-7-5$ & 0 & 0 & 0 & 1 & 0 & 0 & 0 & 0 & 0 & 0 & 0 & 0 & 1 \\
\hline $8-5-11-6-5$ & 0 & 0 & 1 & 0 & 0 & 0 & 0 & 0 & 0 & 0 & 0 & 0 & 1 \\
\hline $8-5-11-7-5$ & 0 & 0 & 1 & 0 & 0 & 0 & 0 & 0 & 0 & 0 & 0 & 0 & 1 \\
\hline $9-5-12-7-5$ & 0 & 1 & 0 & 0 & 0 & 0 & 0 & 0 & 0 & 0 & 0 & 0 & 1 \\
\hline Frequency $^{\mathrm{c}}$ & 4 & 4 & 5 & 4 & 1 & 1 & 2 & 1 & 3 & 1 & 1 & 1 & \\
\hline
\end{tabular}

a Refers to the frequency of Phylotype II R. solanacearum sequevar 1 strains within the 14 haplotypes across the northern, western, southern, and eastern provinces of Rwanda.

b Only 73 RSSC sequevar 1 strains were included in the multilocus VNTR (variable number of tandem repeats) sequence analysis.

${ }^{\mathrm{c}}$ Frequency of TRST haplotype profiles within a district. 
were most susceptible to the Rw7 and Rw15, with incidence being in the range of 66 to $77 \%$ for Rw7 and 42 to $61 \%$ for Rw 15 at 4 DAI, and increased to $100 \%$ by 12 and 16 DAI, along with 100 DSI at 28 and 32 DAI for Rw7 and Rw15, respectively. Kazeneza was highly susceptible to Rw7 but moderately to Rw15, whereas Cruza showed some tolerance for Rw15 and Rw7, and Twihaze only for Rw15. In contrast, bacterial wilt incidence and severity remained very low for Rw62, appearing first at 12 DAI and increasing over time to a maximum of 36 DSI on Victoria, Kinigi, and Kirundo, and remained in the range of 7 to $9 \%$ BWI and 5 to 6 DSI among Cruza, Twihaze, and Kazeneza until 44 DAI.

There was a cultivar $\times$ isolate interaction $(P=0.001)$ for AUDPC, so the reaction of each cultivar to the individual strains was further investigated. Mean AUDPCs differed among Rw7, Rw15, and Rw62, with Rw7 being highly aggressive, Rw15 intermediate, and Rw62 the least (Fig. 4). Cultivars showed a differential reaction to phylotypes: Victoria, Kinigi, and Kirundo were highly susceptible to Rw7, intermediate to Rw15, and least to Rw62. The lowest value of AUDPC was recorded in Cruza across all phylotypes tested; Twihaze and Cruza had similar reaction against RW15, and same was true for Victoria, Kinigi, Kirundo, Twihaze, Kazeneza, and Cruza against Rw62. Based on the assessment, Cruza remained the least susceptible cultivar followed by Twihaze and Kazeneza whereas Victoria, Kirundo, and Kinigi were highly susceptible.
Latent infection by RSSC strains were positive for all tested asymptomatic plants; a typical Ralstonia colony (irregular in shape, white to cream color, slimy with pink color in the center) grew on $\mathrm{CPG}$ media after $28 \mathrm{~h}$ incubation at $28^{\circ} \mathrm{C}$. RSSC phylotype level testing also confirmed the latent infection of respective strains (data not shown).

\section{Discussion}

The phylotype II and phylotype III strains of $R$. solanacearum species complex (RSSC) were distributed in all surveyed districts and caused bacterial wilt of potato in Rwanda. Our study has confirmed the presence of phylotype II strains in 12 out of 14 districts representing all the potato growing provinces of Rwanda. This widespread distribution of the potato bacterial wilt pathogen in Rwanda is consistent with a recent report of Uwamahoro et al. (2018). The finding of phylotype II sequevar 1 strains at higher rates in all 12 districts suggest that phylotype II sequevar 1 strains is currently endemic in the country and is the most epidemic strain of potato bacterial wilt. Although phylotype II and I strains were reported earlier from Rwanda (Butare 1987; Lepoint and Maraite 2002; Poussier et al. 2000b), this is the first report of phylotype III strains from Rwanda, and the first to show the epidemiologically important strains among phylotype II sequevar 1 in bacterial wilt of potato in Rwanda.

Phylotype III strains appear to be epidemiologically not important in Rwanda with the very low distribution rate $(4.21 \%, n=4)$.

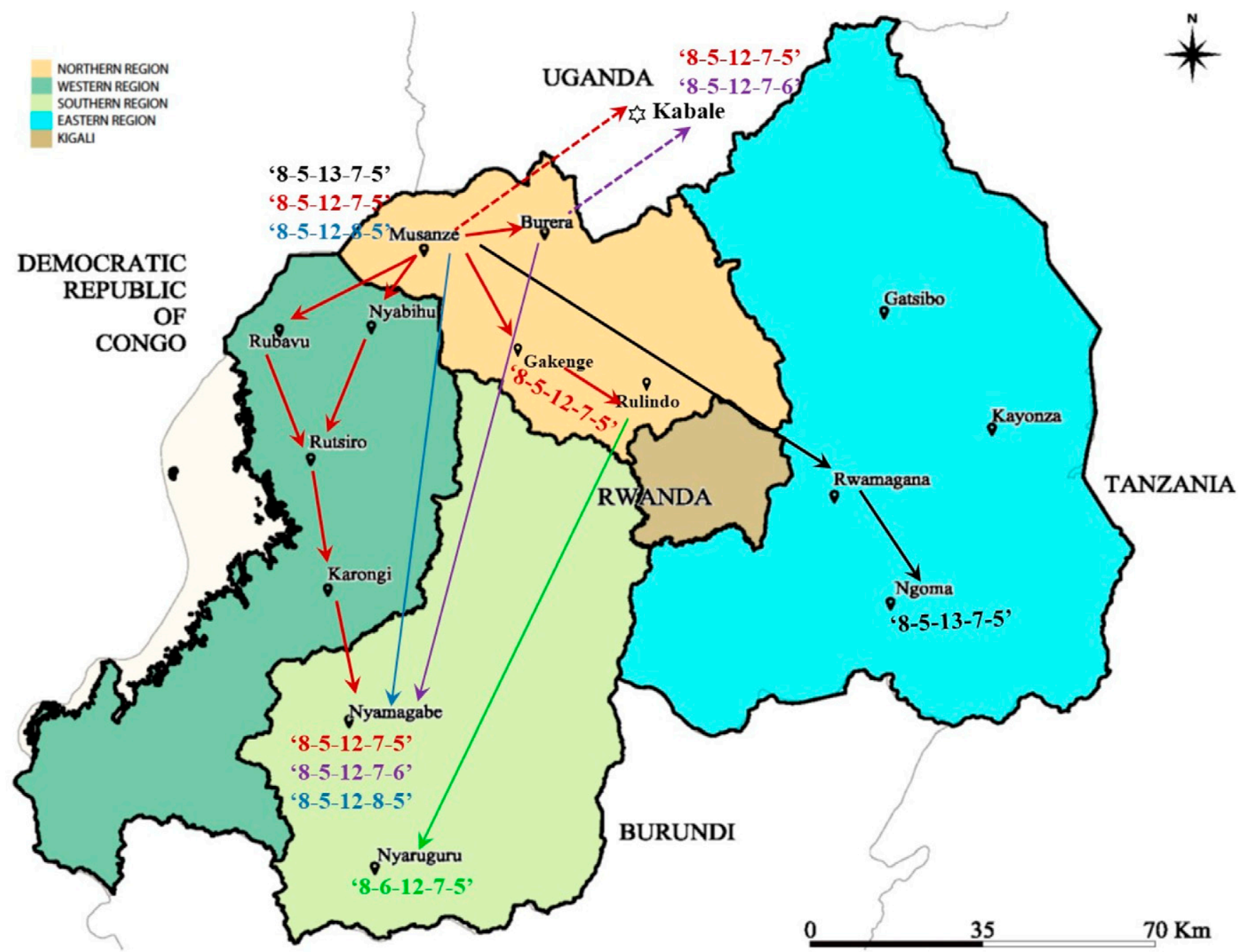

Fig. 2. Tandem repeat sequence haplotype localization of epidemiologically important phylotype II Ralstonia solanacearum sequevar 1 strains in Rwanda. Red arrows indicate the main seed potato distribution pattern from Musanze district of the northern province to Nyabihu, Rubavu, Rutsiro, and Karongi districts of the western province, and to Nyamagabe and Nyaruguru districts of the southern province, whereas the gray line indicates the seed distribution pattern from Musanze to the eastern province. Purple, blue, and brown lines refer to the direct seed distribution from the northern to southern provinces. Red and purple dashed lines indicate the informal seed exchange route from Musanze and Burera districts of the northern province to Kabale district of Uganda. Inference is made that the TRST ' $8-5-12-7-5$ ' haplotype is being distributed along with latently infected seed potato from Musanze district to the western and southern provinces; however, from the restriction of TRST '8-5-12-7-6' haplotype to only Kabale district in Uganda (Abdurahman et al. 2019), it is inferred that the strain originated from Burera district of Rwanda. 
Although phylotype III strains are believed to originate from Africa, and are endemic in Cameroun (Mahbou Somo Toukam et al. 2009), Tanzania (Kanyagha et al. 2018), Burundi (Elphinstone 2005; Fegan and Prior 2005), Ivory Coast (N'Guessan et al. 2013), and the Democratic Republic of Congo (DRC) (Elphinstone 2005; Fegan and Prior 2005), our finding is consistent with phylotype III reports from Cameroun (Mahbou Somo Toukam et al. 2009), Ivory Coast (N'Guessan et al. 2013), and Uganda (Abdurahman et al. 2019) where a minority of potato-infecting strains were phylotype III (18, 1.8 , and $1.5 \%$, respectively). Thus, phylotype III strains appear to be occasional opportunistic pathogens of potato, with very limited epidemic significance.

The finding of phylotype III strains in the northern (Musanze district), eastern (Kayonza district), and sourthern (Nyaruguru district) provinces of Rwanda but not in the main seed potato-producing districts of the western province indicate that the strains were localized to those districts and are of only local importance. The localized existence of Rw15 in Musanze district bordering the DRC, Rw62 in Kayonza district bordering Tanzania, and Rw49 in Nyaruguru district bordering Burundi also indicates their introduction via latently infected seed, as there is an active informal seed and ware potato trade between these bordering countries.

Phylotype II sequevar 1 strains are thought to be clonal (monomorphic) lineages causing potato bacterial wilt mainly in the highland tropics (Wicker et al. 2012). The lower diversity index of phylotype II sequevar 1 strains from Rwanda compared with Ethiopia (Abdurahman et al. 2017) and Uganda (Abdurahman et al. 2019) indicates a more clonal population structure of sequevar 1 strains from Rwanda.

Seed potatoes are mainly produced in the northern (Muzanze and Burera districts) and western (Nyabihu and Rubavu districts) provinces followed by the southern province (Ferrari et al. 2018), with primary seed being produced in the Musanze district. Newly emerging potato-producing districts of the eastern province also receive seed from Musanze. Mapping of the most frequent TRST haplotype of phylotype II sequevar 1 strains of this study appears to be consistent with the seed movement from the northern province to the western, southern, and eastern provinces, indicating the strain movement via latently infected seed. It is also likely that Rw61 strain in the Kayonza district of the eastern province might have been introduced with latently infected seed from Musanze as Rw15 and Rw61 clustered together with $96 \%$ bootstrap value, which is also supported by the confirmation of latent infections in our controlled pathogenicity experiments. However, additional TRST typing of Rw15 and Rw61 strains is required to confirm the epidemiological inferences.

TRST haplotype 8-5-12-7-5 was the most widespread and frequent haplotype in Rwanda and was also the most widespread haplotype found in Uganda (Rs-TRST 5; Abdurahman et al. 2019) and Ethiopia (MLVA profile P2; Abdurahman et al. 2017). Another TRST haplotype 8-5-12-7-6 found in the Burera district of Rwanda was also identical to Rs-TRST3, which was limited to the Kabale district of Uganda (Rs-TRST3; Abdurahman et al. 2019). Informal seed and ware potato exchange between Kabale-Kisoro districts of Uganda and the bordering Musanze-Burera districts in Rwanda is very common, usually growing the same potato cultivars (mainly Cruza, Kinigi, Kirundo, and Victoria) along the neighboring districts. It is expected that the same pathogenic strains are disseminated via

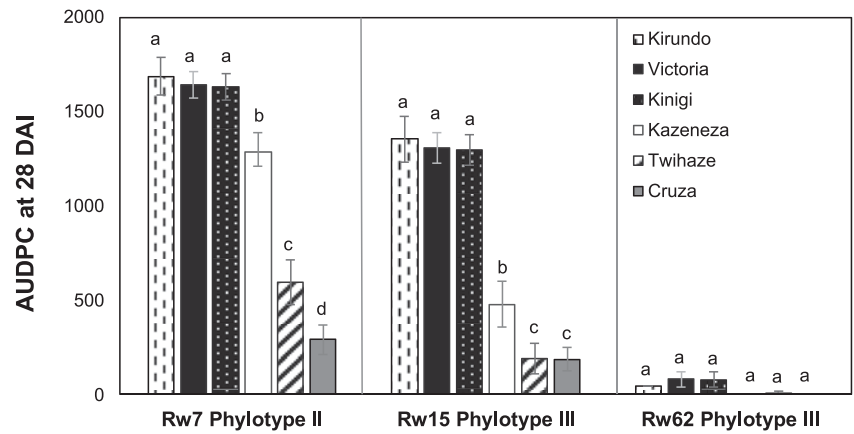

Fig. 4. Area under bacterial wilt severity progress curves (AUDPC) of Rwandan potato cultivars Victoria, Cruza, Twihaze, Kazeneza, Kinigi, and Kirundo inoculated with Rw7 (phylotype II Ralstonia solanacearum), and Rw15 and Rw62 (phylotype III $R$. pseudosolanacearum) strains. Reaction of cultivars to the individual strains is presented in the same graph. Bars with the same letter above do not differ, based on Tukey's test at $P=0.001$. Capped lines represent \pm standard error.
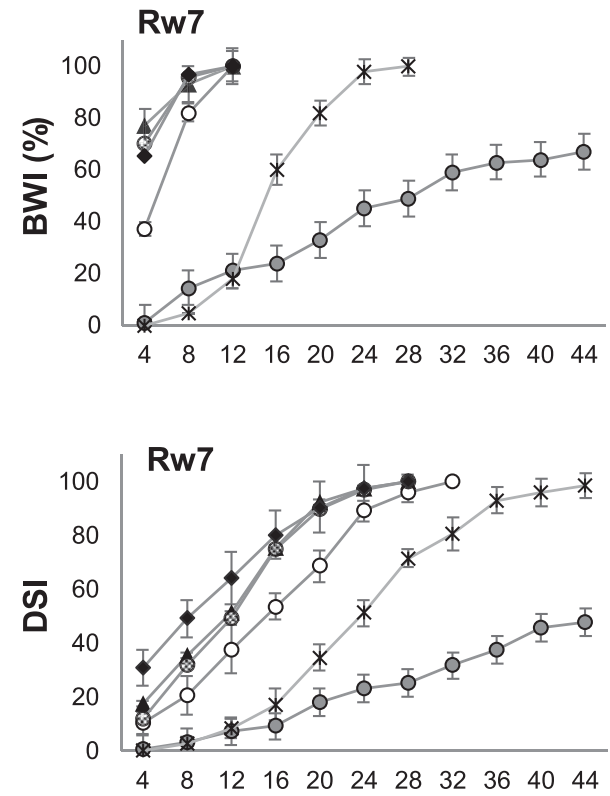

Days after inoculation
Rw15
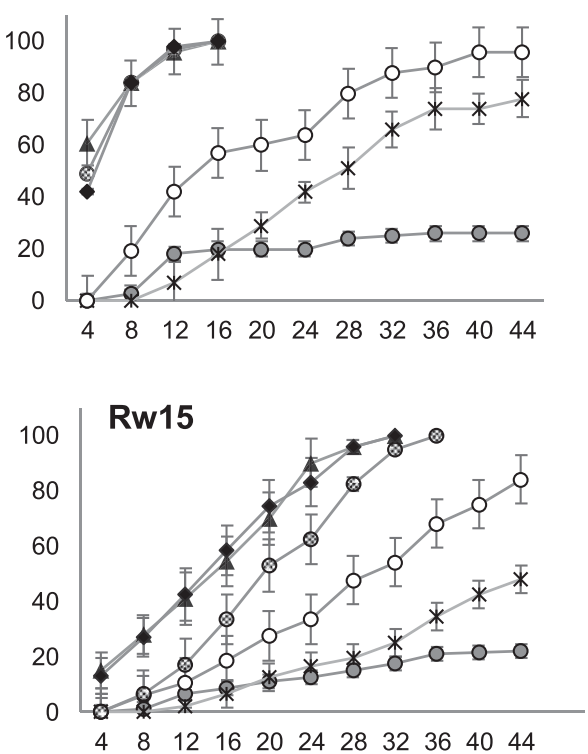

Days after inoculation
Rw62

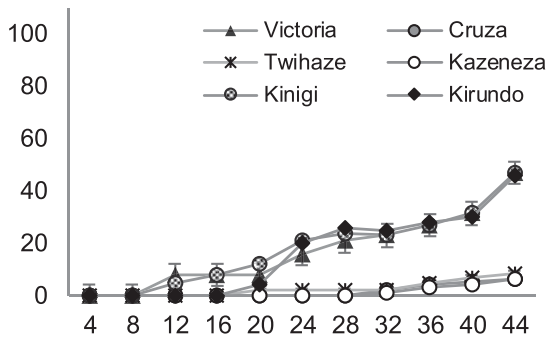

Rw62

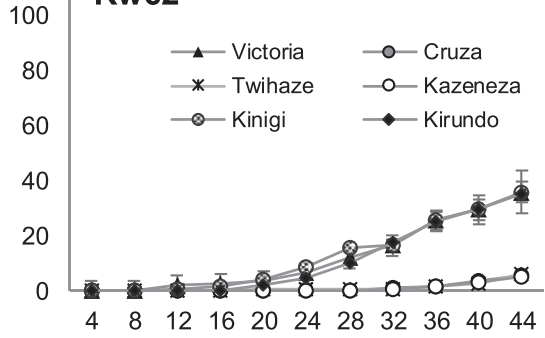

Days after inoculation

Fig. 3. Bacterial wilt incidence (BWI) and wilting severity index (DSI) of Rwandan potato cultivars Victoria, Cruza, Twihaze, Kazeneza, Kinigi, and Kirundo assessed from 4 to 44 days after inoculation (DAl) with Rw7 (phylotype II Ralstonia solanacearum), and Rw15 and Rw62 (phylotype III R. pseudosolanacearum) strains. Capped lines represent \pm standard error. 
latently infected seed along and within international borders, which is evidenced by the unique 8-5-12-7-6 and 8-5-12-7-5 haplotypes found in Uganda and Rwanda. On the other hand, the 8-5-12-8-5 haplotype shared an identical profile from Ethiopia (P4; Abdurahman et al. 2017). A single strain with 8-5-13-7-5 haplotype isolated from Musanze, Ngoma, and Rwamagana districts appears to have the same TRST profile with many strains reported in Madagascar by Ravelomanantsoa et al. (2018), including strain JT573 reportedly isolated from potatoes in Rwanda except for Locus IPO100. This discrepancy may be explained by the fact that the work of Ravelomanantsoa et al. (2018) depends on amplicon size whereas our analysis is based on sequence repeats with defined start and end sequences. As an example, if the post repeat sequence is set as GCCGGGTGGATT accounting for additional GGTGCCC or AGTGTGC as repeat sequences, the data at locus IPO100 appears to agree.

None of the six potato test cultivars was found to have complete resistance to the tested strains, but the level of susceptibility varied among cultivars. The reaction of Victoria, Kirundo, Twihaze, and Cruza to Rw7 (phylotype II) of this study is consistent with the recent report of Uwamahoro et al. (2020). The three strains used differed in their aggressiveness on the six tested potato cultivars. With the less aggressive strain Rw62 the disease onset and progress were delayed irrespective of the cultivar, but this effect was more evident in the cultivars with some level of tolerance (Fig. 3 and 4). For example, the reduction in AUDPC between Rw7 and Rw15 was only $20 \%$ in susceptible cultivars: Kirundo, Victoria, and Kinigi; it was 35, 62, and $68 \%$ for Cruza, Twihaze, and Kazeneza, respectively. This indicates that differences in disease severity caused by different strains are more strongly associated with intrinsic differences in the strains' aggressiveness, and also influenced by specific host cultivar interactions, resistance apparently being proportionally more effective against less aggressive strains. Further research including the predominant phylotype II strains with multiple TRST haplotypes profile is needed to confirm pathogenicity diversity among the phylotype II strains.

It is important to note that all the asymptomatic plants had the latent infection. For this reason, the International Potato Center (CIP) for many years avoided developing resistant cultivars that were not completely immune to the pathogen. However, considering even susceptible cultivars can sometimes escape infection and could be latent carriers of disease, this strategy can be called into question. Nevertheless, breeding for resistance to bacterial wilt has made little progress due to extremely low levels of heritability and complex potato genetics, and Cruza is still the most resistant cultivar available since 40 years after its initial development (French et al. 1998; Gutarra et al. 2015; Uwamahoro et al. 2020). This is the first report to present the reaction of commonly grown potato cultivars to phylotype III strains in Rwanda. The results of pathogenicity assays suggest that phylotype III strains could cause significant disease in Rwanda, although their number and distribution rate is very low compared with phylotype II strains. Additional research is required to confirm the differential reaction of these cultivars under field conditions.

Smallholder farmers in Rwanda ranked bacterial wilt control as the highest priority (NISR 2018), and reported Kinigi, Kirundo, Victoria, and Twihaze among the highly susceptible cultivars to bacterial wilt (Muhinyuza et al. 2012), which is consistent with our results. Our study also confirmed a very high rate of RSSC strains in the primary potato-producing districts of the northern and western provinces (58.95\% and $22.11 \%$, respectively), moderate rate in the secondary potato-producing districts of the southern province $(14.74 \%)$, and very low rate in the eastern province $(4.21 \%)$ where potato is currently a minor crop and accounts for only $1.5 \%$ of the total area under potato production in the country (NISR 2018). The presence and widespread distribution of RSSC strains in all study districts confirms the presence of RSSC strains in Rwanda for many years (Van der Zaag 1985), and the pathogen could continue to be spread through latently infected seed potatoes to the rest of the country (Muhinyuza et al. 2012).

From this study, we conclude that bacterial wilt of potato is widespread in the major potato growing districts of Rwanda and is encroaching into the newly emerging potato producing districts of the eastern province. Although this study confirmed the presence of phylotype II and phylotype III strains in Rwanda, only phylotype II sequevar 1 strains are important for potato production. TRST 8-512-7-5 haplotype strains were epidemiologically more important in the country, likely being spread via latently infected seed. No immunity or absolute resistance to phylotype II and phylotype III strains is available in the tested cultivars, but the level of susceptibility varied significantly among cultivars across the tested strains. More research is needed to determine whether the same gene or different genes are responsible for partial resistance to different phylotypes, and whether the tolerant cultivars identified here are also tolerant to other RSSC strains. Meanwhile, Cruza with less susceptibility to the disease could be recommended in the fields with the history of bacterial wilt.

\section{Acknowledgments}

The authors would like to thank Rwanda Agriculture and Animal Resources Development Board (RAB), Musanze Research Station, for selecting survey districts and conducting the bacterial wilt surveys, and Benson Kisinga for the mapping exercise. This research was undertaken as part of the CGIAR Research Program on Roots, Tubers and Bananas (RTB).

\section{Literature Cited}

Abdurahman, A., Griffin, D., Elphinstone, J. G., Struik, P. C., Schulz, S., SchulteGeldermann, E., and Sharma, K. 2017. Molecular characterization of Ralstonia solanacearum strains from Ethiopia and tracing potential source of bacterial wilt disease outbreak in seed potatoes. Plant Pathol. 66:826-834.

Abdurahman, A., Parker, M., Kreuze, J., Elphinstone, J., Struik, P., Kigundu, A., Arengo, E., and Sharma, K. 2019. Molecular epidemiology of Ralstonia solanacearum species complex strains causing bacterial wilt of potato in Uganda. Phythopathology 109:1922-1931.

Álvarez, B., Lopez, M., and Biosca, G. 2008. Survival strategies and pathogenicity of Ralstonia solanacearum phylotype II subjected to prolonged starvation in environmental water microcosms. Microbiology 154:3590-3598.

Butare, J. B. 1987. Incidence des traitements aux extraits végétaux sur l'épidemiologie de la bacteriose vasculaire de la pomme de terre (due à Pseudomonas solanacearum E.F. SMITH) au Rwanda. Memoire de fin d'études. University National du Rwanda, Butare, Rwanda.

Campbell, C. L., and Madden, L. V. 1990. Introduction to Plant Disease Epidemiology. John Wiley \& Sons, New York.

Chesneau, T., Maignien, G., Boyer, C., Ch'eron, J. J., Roux-Cuvelier, M., Vanhuffel, L., Poussier, S., and Prior, P. 2018. Sequevar diversity and virulence of Ralstonia solanacearum phylotype I on Mayotte Island (Indian Ocean). Front. Plant Sci. 8:2209.

Davey, J. W., Hohenlohe, P. A., Etter, P. D., Boone, J. Q., Catchen, J. M., and Blaxter, M. L. 2011. Genome-wide genetic marker discovery and genotyping using next-generation sequencing. Nat. Rev. Genet. 12:499-510.

Demo, P., Lemaga, B., Kakuhenzire, R., Schulz, S., Borus, D., Barker, I., Woldegiorgis, G., Parker, M. L., and Schulte-Geldermann E. 2015. Strategies to improve seed potato quality and supply in Sub-Saharan Africa: experience from interventions in five countries. Page 155 in: Potato and sweetpotato in Africa: Transforming the value chains for food and nutrition security. CABI, Wallingford, UK.

Devaux, A., Michelante, D., and Bicamumpaka, M. 1987. Combination of rotation and resistance to control bacterial wilt (Pseudomonas solanacearum) in Rwanda. Pages 100-101 in: X Triennial Conference Abstracts. European Association for Potato Research, Leuven, Belgium.

Elphinstone, J. G. 2005. The current bacterial wilt situation: a global overview. Pages 9-28 in: Bacterial Wilt Disease and the Ralstonia solanacearum Species Complex. C. Allen, P. Prior, and A. C. Hayward, eds. APS Press, Saint Paul, MN.

FAOSTAT. 2017. http://www.fao.org/faostat/en/\#data/QC. Accessed 21 February 2018.

Fegan, M., and Prior, P. 2005. How complex is the Ralstonia species complex. Pages 449-461 in: Bacterial Wilt Disease and the Ralstonia solanacearum Species Complex. C. Allen, P. Prior, and A. C. Hayward, eds. APS Press, Saint Paul, MN.

Ferrari, L., Fromm, I., Scheidegger, U., and Muhire, A. 2018. Formal or informal? Analysis of the potato seed system in Rwanda. J. Agric. Res. (Lahore) 3: 000206.

French, E., Anguiz, R., and Aley, P. 1998. The usefulness of potato resistance to Ralstonia solanacearum, for the integrated control of bacterial wilt. Bacterial wilt disease. Pages 381-385 in: Bacterial Wilt Disease. P. Prior, C. Allen, and J. Elphinstone, eds. Springer, Berlin, Heidelberg, Germany.

Genin, S. 2010. Molecular traits controlling host range and adaptation to plants in Ralstonia solanacearum. New Phytol. 187:920-928.

Gillings, M. R., and Fahy, P. 1994. Genomic fingerprinting: towards a unified view of the Pseudomonas solanacearum species complex. Pages 95-112 in: Bacterial Wilt: The Disease and its Causative Agent, Pseudomonas solanacearum. A. C. Hayward and G. L. Hartman, eds. CAB International, Wallingford, UK. 
Guidot, A., Prior, P., Schoenfeld, J., Carrere, S., Genin, S., and Boucher, C. 2007. Genomic structure and phylogeny of the plant pathogen Ralstonia solanacearum inferred from gene distribution analysis. J. Bacteriol. 189:377-387.

Gutarra, L., Kreuze, J., Lindqvist-Kreuze, H., and De Mendiburu, F. 2015. Variation of resistance to different strains of Ralstonia solanacearum in highland tropics adapted potato genotypes. Am. J. Potato Res. 92:258-265.

Haubold, B., and Hudson, R. 2000. LIAN 3.0: Detecting linkage disequilibrium in multilocus data. Bioinformatics 16:847-849.

Hayward, A. C. 1974. Latent infections by bacteria. Annu. Rev. Phytopathol. 12: 87-97.

Horita, M., and Tsuchiya, K. 2001. Genetic diversity of Japanese strains of Ralstonia solanacearum. Phythopathology 91:399-407.

Hunter, P., and Gaston, M. 1988. Numerical index of the discriminatory ability of typing systems: An application of Simpson's index of diversity. J. Clin. Microbiol. 26:2465-2466.

Janse, D. 1996. Potato brown rot in Western Europe - history, present occurrence and some remarks on possible origin, epidemiology and control strategies. Bull. OEPP/EPPO Bull. 26:679-695.

Kanyagha, H., Rotondo, F., Testen, L. A., and Miller, A. S. 2018. Survey and characterization of Ralstonia solanacearum in solanaceous crops in Tanzania. Plant Health in Global Economy. International Congress of Plant Pathology (ICPP) Boston July 29-August 3, conference abstract.

Kathiresan, A. 2011. Strategies for sustainable crop intensification in Rwanda: shifting focus from producing enough to producing surplus. Ministry of Agriculture and Animal Resources, Kigali, Rwanda.

Lepoint, P., and Maraite, H. 2002. Status of bacterial wilt in the Rwandan potato seed multiplication scheme. Bachelor thesis, Université Catholique de Louvain, Belgium.

Lund, R. E. 1975. Tables for an approximate test for outliers in linear models. Techometrics 17:473-476.

Mahbou Somo Toukam, G., Cellier, G., Wicker, E., Guilbaud, C., Kahane, R., Allen, C., and Prior, P. 2009. Broad diversity of Ralstonia solanacearum strains in Cameroon. Plant Dis. 93:1123-1130.

Mansfield, J., Genin, S., Magori, S., Citovsky, V., Sriariyanum, M., Ronald, P., Dow, X., Verdier, V., Beer, S., Machado, M., Toth, I., and Salmond, G. 2012. Top 10 plant pathogenic bacteria in molecular plant pathology. Mol. Plant Pathol. 13:614-629.

Mpyisi, E., Weber, M., Shingiro, E., and Loveridge, S. 2003. Changes in allocation of land holdings, production and farm size in the Rwandan smallholder sector over the period 1984/1990 to 2002. Agricultural Policy Synthesis 6E. https:// core.ac.uk/download/pdf/6675045.pdf

Muhinyuza, J. B., Shimelis, H., Melis, R., Sibiya, J., and Nzaramba, M. N. 2012. Participatory assessment of potato production constraints and trait preferences in potato variety development in Rwanda.Int. J. Develop. Sustain. 1:358-380.

Muthoni, J., Shimelis, H., Melis, R., and Kinyua, Z. 2014. Response of potato genotypes to bacterial wilt caused by Ralstonia solanacearum (Smith) (Yabuuchi et al.) in the tropical highlands. Am. J. Potato Res. 91:215-232.

Mutimawurugo, M. C., Wagara, I. N., Muhinyuza, J. B., and Ogweno, J. O. 2019. Virulence and characterization of isolates of potato bacterial wilt caused by Ralstonia solanacearum (Smith) in Rwanda.African J. Agric. Res. 14:311-320.

N'Guessan, C., Brisse, S., Le Roux-Nio, A., Poussier, S., Koné, D., and Wicker, E. 2013. Development of variable number of tandem repeats typing schemes for Ralstonia solanacearum, the agent of bacterial wilt, banana Moko disease and potato brown rot. J. Microbiol. Methods 92:366-374.

Nei, M. 1978. Estimation of average heterozygosity and genetic distance from a small sample of individuals. Genetics 89:583-590.

NISR. 2018. Seasonal Agricultural Survey, 2017. National Institute of Statistics of Rwanda, Kigali, Rwanda. https://microdata.statistics.gov.rw/index.php/catalog/ 88/download/819

Opina, N., Tavner, F., Hollway, G., Wang, F., Li, H., Maghirang, R., Fegan, M., Hayward, C., Krishnapillai, V., Hong, F., Holloway, W., and Timmis, N. 1997. A novel method for development of species and strain-specific DNA probes and PCR primers for identifying Burkholderia solanacearum (formerly Pseudomonas solanacearum). Asia Pac. J. Mol. Biol. Biotechnol. 5:19-30.

Parkinson, N., Bryant, R., Bew, J., Conyers, C., Stones, R., Alcock, M., and Elphinstone, J. 2013. Application of variable-number tandem-repeat typing to discriminate Ralstonia solanacearum strains associated with English watercourses and disease outbreaks. Appl. Environ. Microbiol. 79:6016-6022.

Poussier, S., Prior, P., Luisetti, J., Hayward, C., and Fegan, M. 2000a. Partial sequencing of the hrpB and endoglucanase genes confirms and expands the known diversity within the Ralstonia solanacearum species complex. Syst. Appl. Microbiol. 23:479-486.

Poussier, S., Trigalet Demery, D., Vandewalle, P., Goffinet, B., Luisetti, J., and Trigalet, A. 2000b. Genetic diversity of Ralstonia solanacearum as assessed by PCR-RFLP of the hrp gene region, AFLP and 16S rRNA sequence analysis, and identification of an African subdivision. Microbiology 146: $1679-1692$

Ravelomanantsoa, S., Verniere, C., Rieux, A., Costet, L., Chiroleu, F., Arribat, S Cellier, G., Pruvost, O., Poussier, S., Robene, I., Guerin, F., and Prior, P. 2018. Molecular epidemiology of bacterial wilt in the Madagascar highlands caused by Andean (phylotype IIB-1) and African (phylotype III) brown rot strains of the Ralstonia solanacearum species complex. Front. Plant Sci. 8:2258.

Safni, I., Cleenwerck, I., De Vos, P., Fegan, M., Sly, L., and Kappler, U. 2014. Polyphasic taxonomic revision of the Ralstonia solanacearum species complex: proposal to emend the descriptions of Ralstonia solanacearum and Ralstonia syzygii and reclassify current $R$. syzygii strains as Ralstonia syzygii subsp. syzygii subsp. nov., $R$. solanacearum phylotype IV strains as Ralstonia syzygii subsp. indonesiensis subsp. nov., banana blood disease bacterium strains as Ralstonia syzygii subsp. celebesensis subsp. nov. and $R$ solanacearum phylotype I and III strains as Ralstonia pseudosolanacearum sp. nov. Int. J. Syst. Evol. Microbiol. 64:3087-3103.

Selander, R., Caugant, D., Ochman, H., Musser, J., Gilmour, M., and Whittam, T. 1986. Methods of multilocus enzyme electrophoresis for bacterial population genetics and systematics. Appl. Environ. Microbiol. 51:873-884.

Sola, C., Filliol, I., Legrand, E., Lesjean, S., Locht, C., Supply, P., and Rastogi, N. 2003. Genotyping of the Mycobacterium tuberculosis complex using MIRUs: association with VNTR and spoligotyping for molecular epidemiology and evolutionary genetics. Infect. Genet. Evol. 3:125-133.

Strelkov, S. E., Tewari, J. P., Smith, E., and Smith-Degenhardt, E. 2006 Characterization of Plasmodiophora brassicae populations from Alberta, Canada. Can. J. Plant Pathol. 28:467-474.

Stulberg, M. J., and Huang, Q. 2016. A computer program for fast and easy typing of a partial endoglucanase gene sequence into genospecies and sequevars $1 \& 2$ of the Ralstonia solanacearum species complex. J. Microbiol. Methods 123: 101-107.

Tamura, K., Stecher, G., Peterson, D., Filipski, A., and Kumar, S. 2013. MEGA6 Molecular evolutionary genetics analysis version 6.0. Mol. Biol. Evol. 30: $2725-2729$

Uwamahoro, F., Berlin, A., Bucagu, C., Bylund, H., and Yuen, J. 2018. Potato bacterial wilt in Rwanda: occurrence, risk factors, farmers' knowledge and attitudes. Food Secur. 10:1221-1235.

Uwamahoro, F., Berlin, A., Bucagu, C., Bylund, H., and Yuen, J. 2020. Ralstonia solanacearum causing potato bacterial wilt: host range and varieties's susceptibility in Rwanda. Plant Pathol. 69:559-568.

van Belkum, A., Scherer, S., van Alphen, L., and Verbrugh, H. 1998. Shortsequence DNA repeats in prokaryotic genomes. Microbiol. Mol. Biol. Rev. 62:275-293.

Van der Zaag, P. 1985. Potato production under Pseudomonas solanacearum conditions: sources and management of planting material. Pages 84-88 in: Disease in Asia and South Pacific: Proceedings of an international workshop. Los Banos, Philippines.

van Overbeek, L., Bergervoet, J., Jacobs, F., and van Elsas, J. 2004. The lowtemperature-induced viable-but-nonculturable state affects the virulence of Ralstonia solanacearum biovar 2. Phytopathology 94:463-469.

Weller, S. A., Elphinstone, J. G., Smith, N. C., Boonham, N., and Stead, D. E 2000. Detection of Ralstonia solanacearum strains with a quantitative, multiplex, real-time, fluorogenic PCR (TaqMan) assay. Appl. Environ. Microbiol. 66:2853-2858

Wicker, E., Lefeuvre, P., De Cambiaire, J., Lemaire, C., Poussier, S., and Prior, P. 2012. Contrasting recombination patterns and demographic histories of the plant pathogen Ralstonia solanacearum inferred from MLSA. ISME J. 6 : 961-974 